\title{
SMOOTH FRACTAL INTERPOLATION
}

\author{
M. A. NAVASCUÉS AND M. V. SEBASTIÁN \\ Received 12 December 2005; Revised 5 May 2006; Accepted 14 June 2006
}

Fractal methodology provides a general frame for the understanding of real-world phenomena. In particular, the classical methods of real-data interpolation can be generalized by means of fractal techniques. In this paper, we describe a procedure for the construction of smooth fractal functions, with the help of Hermite osculatory polynomials. As a consequence of the process, we generalize any smooth interpolant by means of a family of fractal functions. In particular, the elements of the class can be defined so that the smoothness of the original is preserved. Under some hypotheses, bounds of the interpolation error for function and derivatives are obtained. A set of interpolating mappings associated to a cubic spline is defined and the density of fractal cubic splines in $\mathscr{H}^{2}[a, b]$ is proven.

Copyright (c) 2006 M. A. Navascués and M. V. Sebastián. This is an open access article distributed under the Creative Commons Attribution License, which permits unrestricted use, distribution, and reproduction in any medium, provided the original work is properly cited.

\section{Introduction}

Fractal interpolation techniques provide good deterministic representations of complex phenomena. Barnsley $[2,3]$ and Hutchinson [8] were pioneers in the use of fractal functions to interpolate sets of data. Fractal interpolants can be defined for any continuous function defined on a real compact interval. This method constitutes an advance in the techniques of approximation, since all the classical methods of real-data interpolation can be generalized by means of fractal techniques (see, e.g., $[5,10,12])$.

Fractal interpolation functions are defined as fixed points of maps between spaces of functions using iterated function systems. The theorem of Barnsley and Harrington (see [4]) proves the existence of differentiable fractal interpolation functions. However, in some cases, it is difficult to find an iterated funcion system satisfying the hypotheses of the theorem, mainly whenever some specific boundary conditions are required (see [4]). In this paper, we describe a very general way of constructing smooth fractal

Hindawi Publishing Corporation Journal of Inequalities and Applications Volume 2006, Article ID 78734, Pages 1-20 DOI 10.1155/JIA/2006/78734 
functions with the help of Hermite osculatory polynomials. The proposed method solves the problem with the help of a classical interpolant. The fractal solution is unique and the constructed interpolant preserves the prefixed boundary conditions. The procedure has a computational cost similar to that of the classical method.

As a consequence of the process, we generalize any smooth interpolant by means of a family of fractal functions. Each element of the class preserves the smoothness and the boundary conditions of the original. Under some hypotheses, bounds of the interpolation error for function and derivatives are obtained. Assuming some additional conditions on the scaling factors, the convergence is also preserved.

In the last section, a set of interpolating mappings associated to a cubic spline is defined, in the general frame of functions whose second derivative has an integrable square. In particular, the density of fractal cubic splines in $\mathscr{H}^{2}[a, b]$ is proven.

\section{Construction of smooth fractal interpolants}

2.1. Fractal interpolation functions. Let $t_{0}<t_{1}<\cdots<t_{N}$ be real numbers, and $I=$ $\left[t_{0}, t_{N}\right] \subset \mathbb{R}$ the closed interval that contains them. Let a set of data points $\left\{\left(t_{i}, x_{i}\right) \in\right.$ $I \times \mathbb{R}: i=0,1,2, \ldots, N\}$ be given. Set $I_{n}=\left[t_{n-1}, t_{n}\right]$ and let $L_{n}: I \rightarrow I_{n}, n \in\{1,2, \ldots, N\}$, be contractive homeomorphisms such that

$$
\begin{gathered}
L_{n}\left(t_{0}\right)=t_{n-1}, \quad L_{n}\left(t_{N}\right)=t_{n}, \\
\left|L_{n}\left(c_{1}\right)-L_{n}\left(c_{2}\right)\right| \leq l\left|c_{1}-c_{2}\right| \quad \forall c_{1}, c_{2} \in I
\end{gathered}
$$

for some $0 \leq l<1$.

Let $-1<s_{n}<1, n=1,2, \ldots, N$, and $F=I \times \mathbb{R}$, let $N$ be continuous mappings, let $F_{n}$ : $F \rightarrow \mathbb{R}$ be given satisfying

$$
\begin{gathered}
F_{n}\left(t_{0}, x_{0}\right)=x_{n-1}, \quad F_{n}\left(t_{N}, x_{N}\right)=x_{n}, \quad n=1,2, \ldots, N \\
\left|F_{n}(t, x)-F_{n}(t, y)\right| \leq\left|s_{n}\right||x-y|, \quad t \in I, x, y \in \mathbb{R} .
\end{gathered}
$$

Now define functions

$$
w_{n}(t, x)=\left(L_{n}(t), F_{n}(t, x)\right) \quad \forall n=1,2, \ldots, N,
$$

and consider the following theorem.

Theorem $2.1[2,3]$. The iterated function system (IFS) $\left\{F, w_{n}: n=1,2, \ldots, N\right\}$ defined above admits a unique attractor $G$. $G$ is the graph of a continuous function $f: I \rightarrow \mathbb{R}$ which obeys $f\left(t_{i}\right)=x_{i}$ for $i=0,1,2, \ldots, N$.

The previous function $f$ is called a fractal interpolation function (FIF) corresponding to $\left\{\left(L_{n}(t), F_{n}(t, x)\right)\right\}_{n=1}^{N} \cdot f: I \rightarrow \mathbb{R}$ is the unique function satisfying the functional equation

$$
f\left(L_{n}(t)\right)=F_{n}(t, f(t)), \quad n=1,2, \ldots, N, t \in I,
$$


or

$$
f(t)=F_{n}\left(L_{n}^{-1}(t), f \circ L_{n}^{-1}(t)\right), \quad n=1,2, \ldots, N, t \in I_{n}=\left[t_{n-1}, t_{n}\right] .
$$

Let $\mathscr{F}$ be the set of continuous functions $f:\left[t_{0}, t_{N}\right] \rightarrow \mathbb{R}$ such that $f\left(t_{0}\right)=x_{0}, f\left(t_{N}\right)=$ $x_{N}$. Define a metric on $\mathscr{F}$ by

$$
d(f, g)=\|f-g\|_{\infty}=\max \left\{|f(t)-g(t)| ; t \in\left[t_{0}, t_{N}\right]\right\} \quad \forall f, g \in \mathscr{F} .
$$

Then $(\mathscr{F}, d)$ is a complete metric space.

Define a mapping $T: \mathscr{F} \rightarrow \mathscr{F}$ by

$$
(T f)(t)=F_{n}\left(L_{n}^{-1}(t), f \circ L_{n}^{-1}(t)\right) \quad \forall t \in\left[t_{n-1}, t_{n}\right], n=1,2, \ldots, N .
$$

Using (2.1)-(2.4), it can be proved that $(T f)(t)$ is continuous on the interval $\left[t_{n-1}, t_{n}\right]$ for $n=1,2, \ldots, N$ and at each of the points $t_{1}, t_{2}, \ldots, t_{N-1} . T$ is a contractive mapping on the metric space $(\mathscr{F}, d)$,

$$
\|T f-T g\|_{\infty} \leq|s|_{\infty}\|f-g\|_{\infty}
$$

where $|s|_{\infty}=\max \left\{\left|s_{n}\right| ; n=1,2, \ldots, N\right\}$. Since $|s|_{\infty}<1, T$ possesses a unique fixed point on $\mathscr{F}$, that is to say, there is $f \in \mathscr{F}_{F}$ such that $(T f)(t)=f(t)$ for all $t \in\left[t_{0}, t_{N}\right]$. This function is the FIF corresponding to $w_{n}$.

The most widely studied fractal interpolation functions so far are defined by the following IFS:

$$
\begin{gathered}
L_{n}(t)=a_{n} t+b_{n}, \\
F_{n}(t, x)=s_{n} x+q_{n}(t)
\end{gathered}
$$

with

$$
a_{n}=\frac{\left(t_{n}-t_{n-1}\right)}{\left(t_{N}-t_{0}\right)}, \quad b_{n}=\frac{\left(t_{N} t_{n-1}-t_{0} t_{n}\right)}{\left(t_{N}-t_{0}\right)} .
$$

$s_{n}$ is called the vertical scaling factor of the iterated function system and $s=\left(s_{1}\right.$, $\left.s_{2}, \ldots, s_{N}\right)$ is the scale vector of the transformation. If $q_{n}(t)$ are linear for $t \in\left[t_{0}, t_{N}\right]$ then the FIF is called affine (AFIF) (see $[2,11]$ ). The cubic FIF (see $[10,13]$ ) is constructed using $q_{n}(t)$ as a cubic polynomial.

In many cases, the data are evenly sampled, then

$$
\begin{aligned}
& h=t_{n}-t_{n-1}, \\
& t_{N}-t_{0}=N h .
\end{aligned}
$$

In the particular case, $s_{n}=0$ for all $n=1,2, \ldots, N$, then

$$
F_{n}(t, x)=q_{n}(t)
$$

and $f(t)=q_{n} \circ L_{n}^{-1}(t)$ for all $t \in I_{n}$. 


\section{Smooth fractal interpolation}

2.2. Differentiable fractal interpolation functions. In this section, we study the construction of smooth fractal interpolation functions. The theorem of Barnsley and Harrington [4] proves the existence of differentiable FIFs and gives the conditions for their existence. We look for IFS satisfying the hypotheses of this theorem.

Theorem 2.2 (Barnsley and Harrington [4]). Let $t_{0}<t_{1}<t_{2}<\cdots<t_{N}$ and $L_{n}(t), n=$ $1,2, \ldots, N$, the affine function $L_{n}(t)=a_{n} t+b_{n}$ satisfying the expressions (2.1)-(2.2). Let $a_{n}=$ $L_{n}^{\prime}(t)=\left(t_{n}-t_{n-1}\right) /\left(t_{N}-t_{0}\right)$ and $F_{n}(t, x)=s_{n} x+q_{n}(t), n=1,2, \ldots, N$, verifying (2.3)-(2.4). Suppose for some integer $p \geq 0,\left|s_{n}\right|<a_{n}^{p}$, and $q_{n} \in \mathscr{C}^{p}\left[t_{0}, t_{N}\right] ; n=1,2, \ldots, N$. Let

$$
\begin{gathered}
F_{n k}(t, x)=\frac{s_{n} x+q_{n}^{(k)}(t)}{a_{n}^{k}}, \quad k=1,2, \ldots, p, \\
x_{0, k}=\frac{q_{1}^{(k)}\left(t_{0}\right)}{a_{1}^{k}-s_{1}}, \quad x_{N, k}=\frac{q_{N}^{(k)}\left(t_{N}\right)}{a_{N}^{k}-s_{N}}, \quad k=1,2, \ldots, p .
\end{gathered}
$$

If $F_{n-1, k}\left(t_{N}, x_{N, k}\right)=F_{n k}\left(t_{0}, x_{0, k}\right)$ with $n=2,3, \ldots, N$ and $k=1,2, \ldots, p$, then

$$
\left\{\left(L_{n}(t), F_{n}(t, x)\right)\right\}_{n=1}^{N}
$$

determines a FIF $f \in \mathscr{C}^{P}\left[t_{0}, t_{N}\right]$ and $f^{(k)}$ is the FIF determined by

$$
\left\{\left(L_{n}(t), F_{n k}(t, x)\right)\right\}_{n=1}^{N}
$$

for $k=1,2, \ldots, p$.

From here on, we consider a uniform partition in order to simplify the calculus. In this case,

$$
a_{n}=\frac{1}{N}
$$

If we consider a generic polynomial $q_{n}$, for instance, the equality proposed in the theorem implies the resolution of systems of equations. Sometimes the system has no solution, mainly whenever some boundary conditions are imposed on the function (see [4]). We will proceed in a different way. In order to define an IFS satisfying Theorem 2.2, we consider the following mappings:

$$
\begin{gathered}
L_{n}(t)=a_{n} t+b_{n}, \\
F_{n}(t, x)=s_{n} x+q_{n}(t),
\end{gathered}
$$

where

$$
q_{n}(t)=g \circ L_{n}(t)-s_{n} b(t),
$$

$g$ is a continuous function satisfying

$$
g\left(t_{i}\right)=x_{i}, \quad i=0,1, \ldots, N,
$$


and $b(t)$ is a real continuous function, $b \neq g$, such that

$$
b\left(t_{0}\right)=x_{0}, \quad b\left(t_{N}\right)=x_{N} .
$$

The IFS satisfies the hypotheses (2.1)-(2.5) of Barnsley's theorem (see [11]). In [11], we proved some properties of this fractal function.

Definition 2.3. Consider $g \in \mathscr{C}(I)$ and a partition of the closed interval $I=\left[t_{0}, t_{N}\right], \Delta$ : $t_{0}<t_{1}<\cdots<t_{N}$. Let $b$ be defined as before and let $s=\left(s_{1}, \ldots s_{N}\right)$ be the scaling vector of the IFS defined by (2.11) and (2.21).

The corresponding FIF $g_{\Delta b}^{s}, g_{b}^{s}, g_{\Delta}^{s}$ or simply $g^{s}$ is called $s$-fractal function of $g$ with respect to the partition $\Delta$ and the function $b$.

THeOREM 2.4 (see [11]). The s-fractal function $g_{b}^{s}$ of $g$ with respect to $\Delta$ and $b$ satisfies the inequality

$$
\left\|g_{b}^{s}-g\right\|_{\infty} \leq \frac{|s|_{\infty}}{1-|s|_{\infty}}\|g-b\|_{\infty},
$$

where $|s|_{\infty}=\max _{1 \leq n \leq N}\left\{\left|s_{n}\right|\right\}$. Besides, $g_{b}^{s}$ interpolates to $g$, that is to say,

$$
g_{b}^{s}\left(t_{n}\right)=g\left(t_{n}\right) \quad \forall n=0,1, \ldots, N .
$$

Consequence 2.5. If $s=0$, then $g_{b}^{s}=g$.

Remark 2.6. By (2.7), for all $t \in I_{n}, n=1,2, \ldots, N$,

$$
g_{b}^{s}(t)=g(t)+s_{n}\left(g_{b}^{s}-b\right) \circ L_{n}^{-1}(t)
$$

The first step is to check which conditions should satisfy $b(t)$ in order to fulfill the hypotheses of the theorem of Barnsley and Harrington.

Let us consider $p \geq 0,\left|s_{n}\right|<1 / N^{p}$, and $q_{n}(t) \in \mathscr{C}^{p}\left[t_{0}, t_{N}\right], n=1,2, \ldots, N$.

The prescribed conditions are

$$
F_{n-1, k}\left(t_{N}, x_{N, k}\right)=F_{n k}\left(t_{0}, x_{0, k}\right)
$$

where $n=2,3, \ldots, N, k=1,2, \ldots, p$.

We have from the assumptions (2.15) of the theorem,

$$
F_{n k}(t, x)=\frac{s_{n} x+q_{n}^{(k)}(t)}{a_{n}^{k}} .
$$

In this particular case,

$$
q_{n}(t)=g \circ L_{n}(t)-s_{n} b(t)
$$

as $L_{n}(t)=(1 / N) t+b_{n}$ and $L_{n}^{\prime}(t)=1 / N=a_{n}$, we have for all $k=0,1, \ldots, p$,

$$
q_{n}^{(k)}(t)=\frac{1}{N^{k}} g^{(k)}\left(L_{n}(t)\right)-s_{n} b^{(k)}(t)
$$


6 Smooth fractal interpolation

so that (2.27) becomes

$$
\begin{aligned}
& N^{k} s_{n-1} \frac{g^{(k)}\left(t_{N}\right)-N^{k} s_{N} b^{(k)}\left(t_{N}\right)}{1-N^{k} s_{N}}-s_{n-1} N^{k} b^{(k)}\left(t_{N}\right) \\
&=N^{k} s_{n} \frac{g^{(k)}\left(t_{0}\right)-N^{k} s_{1} b^{(k)}\left(t_{0}\right)}{1-N^{k} s_{1}}-s_{n} N^{k} b^{(k)}\left(t_{0}\right) .
\end{aligned}
$$

If we consider constant scale factors $s_{n}=s_{1}$ for all $n=1, \ldots, N$,

$$
g^{(k)}\left(t_{N}\right)-b^{(k)}\left(t_{N}\right)=g^{(k)}\left(t_{0}\right)-b^{(k)}\left(t_{0}\right) .
$$

A sufficient condition in order to satisfy this equality is

$$
\begin{aligned}
& b^{(k)}\left(t_{0}\right)=g^{(k)}\left(t_{0}\right), \\
& b^{(k)}\left(t_{N}\right)=g^{(k)}\left(t_{N}\right)
\end{aligned}
$$

for $k=0,1,2, \ldots, p$. In this case, we look for a function $b$ which agrees with $g$ at the extremes of the interval until the $p$ th derivative.

The conditions (2.33) will be satisfied if a Hermite interpolating polynomial $b$ is considered, with nodes $t_{0}, t_{N}$ and $p$ derivatives at the extremes. In this case, (see [14]),

$$
b(t)=H_{g}(t)=\sum_{k=0}^{p} g^{(k)}\left(t_{0}\right) \mathscr{L}_{0 k}(t)+\sum_{k=0}^{p} g^{(k)}\left(t_{N}\right) \mathscr{L}_{N k}(t) .
$$

The functions $\mathscr{L}_{i k}$ are defined by means of intermediate $l_{i k}$, for $i=0, N$ and $0 \leq k \leq p$,

$$
l_{0 k}(t)=\frac{\left(t-t_{0}\right)^{k}}{k !}\left(\frac{t-t_{N}}{t_{0}-t_{N}}\right)^{p+1}, \quad l_{N k}(t)=\frac{\left(t-t_{N}\right)^{k}}{k !}\left(\frac{t-t_{0}}{t_{N}-t_{0}}\right)^{p+1}
$$

so that

$$
\begin{aligned}
& \mathscr{L}_{0 p}(t)=l_{0 p}(t), \\
& \mathscr{L}_{N p}(t)=l_{N p}(t),
\end{aligned}
$$

and for $k=p-1, p-2, \ldots, 0$,

$$
\begin{gathered}
\mathscr{L}_{0 k}(t)=l_{0 k}(t)-\sum_{\nu=k+1}^{p} l_{0 k}^{(v)}\left(t_{0}\right) \mathscr{L}_{0 \nu}(t), \\
\mathscr{L}_{N k}(t)=l_{N k}(t)-\sum_{\nu=k+1}^{p} l_{N k}^{(v)}\left(t_{N}\right) \mathscr{L}_{N \nu}(t) .
\end{gathered}
$$

The mappings $\mathscr{L}_{i k}$ satisfy

$$
\mathscr{L}_{i k}^{(\sigma)}\left(t_{j}\right)= \begin{cases}1 & \text { if } i=j, k=\sigma \\ 0 & \text { otherwise }\end{cases}
$$


The degree of $H_{g}(t)$ is $2 p+1$. The function $g$ is an interpolant of the data such that $g \in \mathscr{C}^{p}$.

According to the theorem of Barnsley and Harrington, the IFS associated with the $k$ th derivative of a FIF is expressed by

$$
\begin{gathered}
L_{n}(t)=\frac{1}{N} t+b_{n}, \\
F_{n k}(t, x)=N^{k} s_{1} x+N^{k} q_{n}^{(k)}(t), \quad k=0,1,2, \ldots, p .
\end{gathered}
$$

In our case,

$$
q_{n}(t)=g \circ L_{n}(t)-s_{1} b(t)
$$

where $b(t)$ is a Hermite interpolating polynomial of degree $2 p+1$ of $g$. The derivatives of $q_{n}(t)$ become

$$
q_{n}^{(k)}(t)=\frac{1}{N^{k}} g^{(k)}\left(L_{n}(t)\right)-s_{1} b^{(k)}(t), \quad k=0,1,2, \ldots, p,
$$

so that the IFS defining the $k$ th derivative of $g_{b}^{s}$ is (2.15),

$$
\begin{gathered}
L_{n}(t)=\frac{1}{N} t+b_{n}, \\
F_{n k}(t, x)=N^{k} s_{1} x+g^{(k)} \circ L_{n}(t)-N^{k} s_{1} b^{(k)}(t), \quad k=0,1,2, \ldots, p,
\end{gathered}
$$

that is to say, the map $q_{n k}$ corresponding to $F_{n k}$ is

$$
q_{n k}(t)=g^{(k)} \circ L_{n}(t)-N^{k} s_{1} b^{(k)}(t), \quad k=0,1,2, \ldots, p,
$$

so that the $k$ th derivative of the $s$-fractal function of $g$ with respect to $s$ and $b, g_{b}^{s}$, agrees with the fractal function of $g^{(k)}$ with respect to the scaling vector $N^{k} s$ and $b^{(k)}$ (Definition 2.3):

$$
\left(g_{b}^{s}\right)^{(k)}=\left(g^{(k)}\right)_{b^{(k)}}^{N^{k} s}, \quad k=0,1,2, \ldots, p .
$$

Proposition 2.7. $\left(g_{b}^{s}\right)^{(k)}$ interpolates to $g^{(k)}$ at the nodes of $\Delta$, for $0 \leq k \leq p$.

Proof. The ordinates of $\left(g_{b}^{s}\right)^{(k)}$ at the extremes of the interval are given in the theorem of Barnsley and Harrington. Applying (2.16), (2.33), and (2.41),

$$
\begin{aligned}
\left(g_{b}^{s}\right)^{(k)}\left(t_{0}\right) & =x_{0, k}=\frac{q_{1}^{(k)}\left(t_{0}\right)}{a_{1}^{k}-s_{1}}=\frac{1}{a_{1}^{k}-s_{1}}\left(\frac{1}{N^{k}} g^{(k)}\left(L_{1}\left(t_{0}\right)\right)-s_{1} b^{(k)}\left(t_{0}\right)\right) \\
& =\frac{1}{1-s_{1} N^{k}}\left(g^{(k)}\left(t_{0}\right)-s_{1} N^{k} b^{(k)}\left(t_{0}\right)\right)=g^{(k)}\left(t_{0}\right) .
\end{aligned}
$$

In the same way,

$$
\left(g_{b}^{s}\right)^{(k)}\left(t_{N}\right)=g^{(k)}\left(t_{N}\right)
$$


Now, applying the fixed point equation (2.26) corresponding to $k$ th IFS at $t_{n}$,

$$
\begin{aligned}
\left(g_{b}^{s}\right)^{(k)}\left(t_{n}\right) & =F_{n k}\left(L_{n}^{-1}\left(t_{n}\right),\left(g_{b}^{s}\right)^{(k)} \circ L_{n}^{-1}\left(t_{n}\right)\right) \\
& =N^{k} s_{1}\left(g_{b}^{s}\right)^{(k)} \circ L_{n}^{-1}\left(t_{n}\right)+g^{(k)}\left(t_{n}\right)-N^{k} s_{1} b^{(k)} \circ L_{n}^{-1}\left(t_{n}\right)=g^{(k)}\left(t_{n}\right)
\end{aligned}
$$

since

$$
\begin{gathered}
L_{n}^{-1}\left(t_{n}\right)=t_{N}, \\
\left(g_{b}^{s}\right)^{(k)}\left(t_{N}\right)=g^{(k)}\left(t_{N}\right)=b^{(k)}\left(t_{N}\right) .
\end{gathered}
$$

The properties of $g_{b}^{s}$ are as the following.

(i) $\left(g_{b}^{s}\right)^{(k)}$ interpolates to $g^{(k)}$ at the nodes of the partition $\Delta$, for $0 \leq k \leq p$.

(ii) $g_{b}^{s}$ may be close to $g$ (choosing suitably the scale vector according to (2.24)).

(iii) $g_{b}^{s}$ preserves the $p$-smoothness of $g$.

(iv) $g_{b}^{s}$ preserves the boundary conditions of $g$.

(v) If $s=0, g_{b}^{s}=g$, that is to say, $g$ is a particular case of $g_{b}^{s}$.

Note. Despite the similarity between $g_{b}^{s}$ and $g$, in general, they do not agree. In fact, if $s \neq 0$ and $b \neq g$, then $g_{b}^{s} \neq g$.

Let us assume that $g_{b}^{s}=g$. If $s_{1} \neq 0$, applying (2.26) for $L_{n}(t) \in I_{n}$,

$$
\begin{gathered}
g \circ L_{n}(t)=g \circ L_{n}(t)+s_{1}(g-b)(t), \\
g(t)=b(t)
\end{gathered}
$$

for all $t \in I$.

2.3. Uniform bounds. In order to bound the distance between $g$ and $g_{b}^{s}$, we consider a theorem of Ciarlet et al. concerning Hermite interpolation.

Given a partition $\Delta: t_{0}<t_{1}<\cdots<t_{N}$ of an interval $\left[t_{0}, t_{N}\right], I_{n}=\left[t_{n-1}, t_{n}\right]$ for $1 \leq n \leq$ $N$, the Hermite function space (see [14]) $H_{\Delta}^{p+1}(p \in N)$ is defined by

$$
H_{\Delta}^{p+1}=\left\{\varphi:\left[t_{0}, t_{N}\right] \longrightarrow \mathbb{R} ; \varphi \in \mathscr{C}^{p}\left[t_{0}, t_{N}\right],\left.\varphi\right|_{I_{n}} \in \mathscr{P}_{2 p+1}\right\}
$$

where $\mathscr{P}_{2 p+1}$ is the space consisting of all polynomials of degree at most $2 p+1$.

Theorem 2.8 (Ciarlet et al. [6]). Let $g \in \mathscr{C}^{r}\left[t_{0}, t_{N}\right]$ with $r \geq 2 p+2$, let $\Delta$ be any partition of $\left[t_{0}, t_{N}\right]$, let $\Delta: t_{0}<t_{1}<\cdots<t_{N}$, and let $\varphi(t)$ be the unique interpolation of $g(t)$ in $H_{\Delta}^{p+1}$, that is, $g^{(l)}\left(t_{n}\right)=\varphi^{(l)}\left(t_{n}\right)$, for all $0 \leq n \leq N, 0 \leq l \leq p$. Then

$$
\left\|g^{(k)}-\varphi^{(k)}\right\|_{\infty} \leq \frac{\|\Delta\|^{2 p+2-k}}{2^{2 p+2-2 k} k !(2 p+2-2 k) !}\left\|g^{(2 p+2)}\right\|_{\infty}
$$

for all $k=0,1, \ldots, p+1$.

In the case in study, we consider a single subinterval of length $T=b-a$. To bound the difference between the $k$ th derivative of $g$ and the $k$ th derivative of $g_{b}^{s}$, we can use 
Theorem 2.4,

$$
\left\|\left(g_{b}^{s}\right)^{(k)}-g^{(k)}\right\|_{\infty}=\left\|\left(g^{(k)}\right)_{b^{(k)}}^{N^{k}}-g^{(k)}\right\|_{\infty} \leq \frac{N^{k}\left|s_{1}\right|}{1-N^{k}\left|s_{1}\right|}\left\|g^{(k)}-b^{(k)}\right\|_{\infty} .
$$

Considering that $b(t)=\varphi(t)$ is the Hermite interpolating polynomial of degree $2 p+$ 1 of $g$, theorem of Ciarlet et al. can be used in order to bound $\left\|g^{(k)}-b^{(k)}\right\|_{\infty}$, so that applying (2.51), (2.52) and considering $g \in \mathscr{C}^{(2 p+2)}$,

$$
\begin{aligned}
\left\|\left(g_{b}^{s}\right)^{(k)}-g^{(k)}\right\|_{\infty} & \leq \frac{N^{k}\left|s_{1}\right|}{1-N^{k}\left|s_{1}\right|}\left\|g^{(k)}-b^{(k)}\right\|_{\infty} \\
& \leq \frac{N^{k}\left|s_{1}\right|}{1-N^{k}\left|s_{1}\right|} \frac{T^{2 p+2-k}}{2^{2 p+2-2 k} k !(2 p+2-2 k) !}\left\|g^{(2 p+2)}\right\|_{\infty}, \quad k=0,1, \ldots, p .
\end{aligned}
$$

2.4. An operator of $\mathscr{C}^{P}(I)$. From here on, we denote by $g^{s}$ the $s$-fractal function of $g \in$ $\mathscr{C} P(I)$ with respect to a fixed partition $\Delta$ of the interval, a scaling vector $s$ with constant coordinates $s_{n}=s_{1}$ for all $n=1,2, \ldots N$ and $b(t)=H_{g}(t)$ defined in the preceding sections.

For fixed $\Delta$, let us consider the operator of $\mathscr{C}^{P}(I)$ which assigns $g^{s}$ to the function $g$,

$$
\mathscr{D}_{p}^{s}(g)=g^{s}
$$

Theorem 2.9. $\mathscr{D}_{p}^{s}$ is a linear, injective, and bounded operator of $\mathscr{C}^{P}(I)$.

Proof. The operator is linear as by (2.26) for all $t \in I_{n}$,

$$
\begin{aligned}
f^{s}(t) & =f(t)+s_{n}\left(f^{s}-H_{f}\right) \circ L_{n}^{-1}(t), \\
g^{s}(t) & =g(t)+s_{n}\left(g^{s}-H_{g}\right) \circ L_{n}^{-1}(t) .
\end{aligned}
$$

Multiplying the first equation by $\lambda$ and the second by $\mu$ and considering that

$$
\lambda H_{f}+\mu H_{g}=H_{\lambda f+\mu g},
$$

the function

$$
\lambda f^{s}+\mu g^{s}
$$

satisfies the equation corresponding to

$$
(\lambda f+\mu g)^{s} .
$$

By the uniqueness of the solution, the linearity is proved.

To prove the injectivity, let us consider that $g^{s}=0$. In this case, for all $t \in I_{n}$ by (2.26),

$$
0=g(t)-s_{1} H_{g} \circ L_{n}^{-1}(t)
$$

but this equation is satisfied by $g(t)=0$ and due to the uniqueness of the solution $g=0$. 
We consider $\mathscr{C}^{p}(I)$ endowed with the norm $\|f\|_{\mathscr{C}^{p}(I)}=\sum_{k=0}^{p}\left\|f^{(k)}\right\|_{\infty}$. Using the definition of $H_{g}(t)(2.34)$,

$$
\begin{gathered}
\left\|H_{g}^{(j)}(t)\right\|_{\infty}=\sup \left|\sum_{k=0}^{p}\left(g^{(k)}\left(t_{0}\right) \mathscr{L}_{0 k}^{(j)}(t)+g^{(k)}\left(t_{N}\right) \mathscr{L}_{N k}^{(j)}(t)\right)\right|, \\
\left\|H_{g}^{(j)}(t)\right\|_{\infty} \leq \sup _{t \in I}\|g\|_{\mathscr{C}^{p}(I)} \sum_{k=0}^{p}\left(\left|\mathscr{L}_{0 k}^{(j)}(t)\right|+\left|\mathscr{L}_{N k}^{(j)}(t)\right|\right) .
\end{gathered}
$$

Let us consider

$$
\lambda_{p}=\sup _{\substack{t \in I \\ 0 \leq j \leq p}} \sum_{k=0}^{p}\left(\left|\mathscr{L}_{0 k}^{(j)}(t)\right|+\left|\mathscr{L}_{N k}^{(j)}(t)\right|\right)
$$

then

$$
\begin{gathered}
\left\|H_{g}\right\|_{\mathscr{C} P(I)} \leq\|g\|_{\mathscr{C} P(I)} \lambda_{p}, \\
\left\|g-H_{g}\right\|_{\mathscr{C} P(I)} \leq\left(\lambda_{p}+1\right)\|g\|_{\mathscr{C} P(I)} .
\end{gathered}
$$

On the other hand, using Theorem 2.4, (2.52), and

$$
\frac{N^{j}\left|s_{1}\right|}{1-N^{j}\left|s_{1}\right|} \leq \frac{N^{p}\left|s_{1}\right|}{1-N^{p}\left|s_{1}\right|}
$$

for $0 \leq j \leq p$, one has

$$
\left\|g^{s}-g\right\|_{\mathscr{C} P(I)} \leq \frac{N^{p}\left|s_{1}\right|}{1-N^{p}\left|s_{1}\right|}\left\|g-H_{g}\right\|_{\mathscr{C} P(I)}
$$

by (2.64) and (2.62),

$$
\begin{gathered}
\left\|g^{s}\right\|_{\mathscr{C}^{P}(I)}-\|g\|_{\mathscr{C}^{P}(I)} \leq \frac{N^{p}\left|s_{1}\right|}{1-N^{p}\left|s_{1}\right|}\left(\lambda_{p}+1\right)\|g\|_{\mathscr{C}^{P}(I)}, \\
\left\|g^{s}\right\|_{\mathscr{C}^{P}(I)} \leq \frac{1+\lambda_{p} N^{p}\left|s_{1}\right|}{1-N^{p}\left|s_{1}\right|}\|g\|_{\mathscr{C}^{p}(I)} .
\end{gathered}
$$

As a consequence, $\mathscr{D}_{p}^{s}$ is bounded and

$$
\left\|\mathscr{D}_{p}^{s}\right\| \leq \frac{1+\lambda_{p} N^{p}\left|s_{1}\right|}{1-N^{p}\left|s_{1}\right|} .
$$

2.5. Convergence in $\mathscr{C} P(I)$. Let $x \in \mathscr{C} P(I)$ be an original function providing the data and let $g_{\Delta_{N}} \in \mathscr{C}^{P}(I)$ be an interpolant of $x$ on the partition $\Delta_{N}$. We consider the fractal function $g_{\Delta_{N}}^{s^{N}}$ of $g_{\Delta_{N}}$ with respect to the partition $\Delta_{N}$, the scale vector $s^{N}$ with constant coordinates, and the function $b$ defined by the equality (2.34). 
THeOREM 2.10. If $x \in \mathscr{C} p(I)$ is an original function and $g_{\Delta_{N}}$ is a p-smooth interpolant of $x$ with respect to the partition $\Delta_{N}$, consider a scaling vector $s^{N}$ with constant coordinates such that $\left|s_{1}^{N}\right|<1 / N^{p}$, then

$$
\left\|x-g_{\Delta_{N}}^{s^{N}}\right\|_{\mathscr{C}^{P}(I)} \leq\left\|x-g_{\Delta_{N}}\right\|_{\mathscr{C}^{P}(I)}+\frac{N^{p}\left|s_{1}^{N}\right|}{1-N^{p}\left|s_{1}^{N}\right|}\left(\lambda_{p}+1\right)\left\|g_{\Delta_{N}}\right\|_{\mathscr{C}^{P}(I)} .
$$

Proof. One has

$$
\left\|x-g_{\Delta_{N}}^{s^{N}}\right\|_{\mathscr{C} P(I)} \leq\left\|x-g_{\Delta_{N}}\right\|_{\mathscr{C}^{P}(I)}+\left\|g_{\Delta_{N}}-g_{\Delta_{N}}^{s^{N}}\right\|_{\mathscr{C}^{P}(I)}
$$

by (2.64),

$$
\left\|x-g_{\Delta_{N}}^{s^{N}}\right\|_{\mathscr{C}^{P}(I)} \leq\left\|x-g_{\Delta_{N}}\right\|_{\mathscr{C}^{P}(I)}+\frac{N^{p}\left|s_{1}^{N}\right|}{1-N^{p}\left|s_{1}^{N}\right|}\left\|g_{\Delta_{N}}-H_{g_{\Delta_{N}}}\right\|_{\mathscr{C}^{p}(I)}
$$

Using (2.62), the result is obtained.

Consequence 2.11. If one considers a scaling vector such that $\left|s_{1}^{N}\right|<1 / N^{p+r}$, for $r>0$ fixed, the fractal interpolant $g_{\Delta_{N}}^{s^{N}}$ converges in $\mathscr{C}_{P} P(I)$ towards the original $x$ if $g_{\Delta_{N}}$ does (as $N$ tends to $\infty)$.

Note. The constant $\lambda_{p}$ does not depend on $\Delta_{N}$ but only on the extremes of the interval.

\section{Fractal cubic splines}

In this section, we study the particular case $p=2$, considering the following IFS:

$$
\begin{gathered}
L_{n}(t)=a_{n} t+b_{n}, \\
F_{n}(t, x)=s_{n} x+q_{n}(t),
\end{gathered}
$$

where $a_{n}=1 / N, s_{n}=s_{1}$ for all $n=1,2, \ldots, N$,

$$
q_{n}(t)=g \circ L_{n}(t)-s_{1} b(t)
$$

where $g$ is a cubic spline with respect to a uniform partition $\Delta_{N}\left(g=\sigma_{\Delta_{N}}\right)$ and $b=H_{g}$ is a Hermite interpolating polynomial satisfying the described conditions (2.33) with $p=2$ $(b(t)$ is a polynomial of degree 5$)$.

We use a classical result of splines in order to find $\mathscr{C}^{2}(I)$ bounds of the interpolation error.

Theorem 3.1 (Hall and Meyer [7]). Let $f \in \mathscr{C}^{4}[a, b]$ and $\left|f^{(4)}(t)\right| \leq L$ for all $t \in[a, b]$. Let $\|f\|_{\infty}=\sup |f(t)|$ when $t \in[a, b]$. Let $\Delta_{N}=\left\{a=t_{0}<t_{1}<\cdots<t_{N}=b\right\}$ be a partition of the interval $[a, b]$, with constant distances between nodes $h=t_{n}-t_{n-1}$. Let $\sigma_{\Delta_{N}}$ be the spline function that interpolates the values of the function $f$ at the points $t_{0}, t_{1}, \ldots, t_{N} \in \Delta_{N}$, being $\sigma_{\Delta_{N}}$ type I or II. Then

$$
\left\|f^{(r)}-\sigma_{\Delta_{N}}^{(r)}\right\|_{\infty} \leq C_{r} L h^{4-r} \quad(r=0,1,2)
$$

with $C_{0}=5 / 384, C_{1}=1 / 24, C_{2}=3 / 8$. The constants $C_{0}$ and $C_{1}$ are optimum. 
Remark 3.2. A spline is type I if its first derivatives at $a$ and $b$ are known. A spline is type II if it can be explicitly represented by its second derivatives at $a$ and $b$.

Theorem 3.3. Let $x(t)$ be a function verifying $x(t) \in \mathscr{C}^{4}\left[t_{0}, t_{N}\right]$ and $\left|x^{(4)}(t)\right| \leq L$ for all $t \in\left[t_{0}, t_{N}\right]$. Let $\sigma_{\Delta_{N}}(t)$ be a cubic spline (with respect to the uniform partition $\Delta_{N}$ ) and let $b(t)$ be a Hermite interpolating polynomial of degree 5 of $\sigma_{\Delta_{N}}$ at the extremes of the interval. Choose a scaling vector with constant coordinates such that $\left|s_{1}^{N}\right|<1 / N^{2}$, then

$$
\left\|x-\sigma_{\Delta_{N}}^{s^{N}}\right\|_{\mathscr{C}^{2}(I)} \leq M_{h}+\frac{N^{2}\left|s_{1}^{N}\right|}{1-N^{2}\left|s_{1}^{N}\right|}\left(\lambda_{2}+1\right)\left(M_{h}+\|x\|_{\mathscr{C}^{2}(I)}\right),
$$

where $M_{h}=C_{0} L h^{4}+C_{1} L h^{3}+C_{2} L h^{2}, C_{0}, C_{1}, C_{2}$ are the constants of Hall and Meyer theorem, $T=t_{N}-t_{0}=N h$, and $\lambda_{2}$ is defined in (2.61).

Proof. Applying Theorem 2.10,

$$
\left\|x-\sigma_{\Delta_{N}}^{s^{N}}\right\|_{\mathscr{C}^{2}(I)} \leq M_{h}+\frac{N^{2}\left|s_{1}^{N}\right|}{1-N^{2}\left|s_{1}^{N}\right|}\left(\lambda_{2}+1\right)\left\|\sigma_{\Delta_{N}}\right\|_{\mathscr{C}^{2}(I)} .
$$

Using now theorem of Hall and Meyer,

$$
\left\|\sigma_{\Delta_{N}}\right\|_{\mathscr{C}^{2}(I)} \leq M_{h}+\|x\|_{\mathscr{C}^{2}(I)}
$$

and the result is obtained.

3.1. Convergence in $\mathscr{H}^{2}[a, b]$. In this subsection, we weaken the hypotheses about the original function $x$. The set

$$
\mathscr{H}^{2}[a, b]=\left\{x:[a, b] \longrightarrow \mathbb{R} ; x^{\prime} \text { absolutely continuous; } x^{\prime \prime} \in L^{2}(a, b)\right\}
$$

is a Hilbert space with respect to the inner product

$$
\langle x, y\rangle=\sum_{j=0}^{2}\left\langle x^{(j)}, y^{(j)}\right\rangle_{L^{2}(a, b)}
$$

where

$$
\langle f, g\rangle_{L^{2}(a, b)}=\left(\int_{a}^{b} f(t) g(t) d t\right)^{1 / 2}
$$

and the norm

$$
\|x\|_{\mathscr{H}^{2}[a, b]}=\left(\sum_{j=0}^{2}\|x\|_{j}^{2}\right)^{1 / 2}
$$

where

$$
\|x\|_{j}=\left(\int_{a}^{b}\left(x^{(j)}(t)\right)^{2} d t\right)^{1 / 2}=\left\|x^{(j)}\right\|_{L^{2}(a, b)} \text { for } j=0,1,2 .
$$


Cubic splines belong to $\mathscr{H}^{2}[a, b]$ and these functions satisfy the following very wellknown properties with respect to the elements of $\mathscr{H}^{2}[a, b]$ (see [1]).

Theorem 3.4. Consider $x \in \mathscr{H}^{2}[a, b]$ and consider a sequence of partitions $\Delta_{N}$ such that $\left\|\Delta_{N}\right\| \rightarrow 0$ as $N \rightarrow \infty$. If $\sigma_{\Delta_{N}}$ is a spline of interpolation of $x$ with respect to the partition $\Delta_{N}$, then $\left\{\sigma_{\Delta_{N}}^{(q)}\right\}$ converges uniformly to $x^{(q)}$ on $I=[a, b]$ for $q=0,1$.

Theorem 3.5. If $x \in \mathscr{H}^{2}[a, b]$ and $\sigma_{\Delta}$ is a spline of interpolation of $x$ with respect to the partition $\Delta$, then

$$
\|x\|_{2}^{2}=\left\|\sigma_{\Delta}\right\|_{2}^{2}+\left\|x-\sigma_{\Delta}\right\|_{2}^{2}
$$

Consequence 3.6. One has

$$
\|x\|_{2}^{2}-\left\|\sigma_{\Delta}\right\|_{2}^{2} \geq 0
$$

Consequence 3.7. If $x \in \mathscr{H}^{2}[a, b]$ is such that $x\left(t_{i}\right)=x_{i}$ at the nodes of the partition $\Delta$ and $\sigma_{\Delta}(t)$ is the cubic spline corresponding to $\left(t_{i}, x_{i}\right)$, then

$$
\int_{a}^{b}\left|\sigma_{\Delta}^{\prime \prime}(t)\right|^{2} d t \leq \int_{a}^{b}\left|x^{\prime \prime}(t)\right|^{2} d t
$$

(property of minimum $\|\cdot\|_{2}$-norm).

Theorem 3.9 states the convergence of fractal cubic splines towards the original function if an additional condition is imposed on the scaling factors. We need a previous result concerning fractal interpolation functions.

LEMma 3.8. For a uniform partition and constant scale factors $s_{n}=s_{1}$, for all $n=1, \ldots, N$,

$$
\left\|f^{s}-f\right\|_{L^{2}(a, b)} \leq \frac{\left|s_{1}\right|}{1-\left|s_{1}\right|}\|f-b\|_{L^{2}(a, b)} .
$$

Proof. According to (2.26), for all $t \in I_{n}$,

$$
\begin{gathered}
f^{s}(t)-f(t)=s_{1}\left(f^{s}-b\right) \circ L_{n}^{-1}(t) \\
\left\|f^{s}-f\right\|_{L^{2}(a, b)}^{2}=\sum_{n=1}^{N} s_{1}^{2} \int_{t_{n-1}}^{t_{n}}\left[\left(f^{s}-b\right) \circ L_{n}^{-1}(t)\right]^{2} d t .
\end{gathered}
$$

The change of variable $\tilde{t}=L_{n}^{-1}(t)$ provides

$$
\begin{gathered}
\left\|f^{s}-f\right\|_{L^{2}(a, b)}^{2}=s_{1}^{2} \sum_{n=1}^{N} a_{n} \int_{a}^{b}\left[\left(f^{s}-b\right)(\tilde{t})\right]^{2} d \tilde{t}, \\
\left\|f^{s}-f\right\|_{L^{2}(a, b)}^{2}=s_{1}^{2}\left\|f^{s}-b\right\|_{L^{2}(a, b) .}^{2}
\end{gathered}
$$


As a consequence,

$$
\begin{aligned}
& \left\|f^{s}-f\right\|_{L^{2}(a, b)}=\left|s_{1}\right|\left\|f^{s}-b\right\|_{L^{2}(a, b)} \leq\left|s_{1}\right|\left(\left\|f^{s}-f\right\|_{L^{2}(a, b)}+\|f-b\|_{L^{2}(a, b)}\right), \\
& \left\|f^{s}-f\right\|_{L^{2}(a, b)} \leq \frac{\left|s_{1}\right|}{1-\left|s_{1}\right|}\|f-b\|_{L^{2}(a, b)} .
\end{aligned}
$$

The next theorem states the convergence of fractal cubic splines towards any original function $x \in \mathscr{H}^{2}[a, b]$ when the partition is refined. A part of the proof can be read in [1, Theorem 3.14.1]. For reason of completeness, we include it here.

Theorem 3.9. Consider $x \in \mathscr{H}^{2}[a, b]$ and let $\left\{\Delta_{N}\right\}$ be a sequence of partitions such that $\Delta_{N} \subseteq \Delta_{N+1}$ and $\left\|\Delta_{N}\right\| \rightarrow 0$ as $N \rightarrow \infty$. Let $s^{N} \in(-1,1)^{N}$ be any sequence of scaling vectors with constant coordinates satisfying $\left|s_{1}^{N}\right|<1 / N^{2+r}$, where $r>0$, then $\left\{\sigma_{\Delta_{N}}^{s^{N}}\right\}$ converges to $x$ in the norm of $\mathcal{H}^{2}[a, b]$.

Proof. As $\Delta_{N} \subseteq \Delta_{N+1}$, the spline $\sigma_{\Delta_{N}}$ interpolates to $\sigma_{\Delta_{N+1}}$ at the nodes of $\Delta_{N}$. Applying the property of minimum $\|\cdot\|_{2}$-norm,

$$
\left\|\sigma_{\Delta_{N}}\right\|_{2} \leq\left\|\sigma_{\Delta_{N+1}}\right\|_{2}
$$

As a consequence, $\left\{\left\|\sigma_{\Delta_{N}}\right\|_{2}\right\}_{N=1}^{\infty}$ is increasing. Besides, $\sigma_{\Delta_{N}}$ is bounded because of (3.13),

$$
\left\|\sigma_{\Delta_{N}}\right\|_{2} \leq\|x\|_{2}
$$

therefore, $\left\{\left\|\sigma_{\Delta_{N}}\right\|_{2}\right\}_{N=1}^{\infty}$ is a convergent sequence. Using the same argument, as $\Delta_{N} \subseteq$ $\Delta_{N+p}$ for all $p \in N$, by (3.12),

$$
\left\|\sigma_{\Delta_{N+p}}-\sigma_{\Delta_{N}}\right\|_{2}^{2}=\left\|\sigma_{\Delta_{N+p}}\right\|_{2}^{2}-\left\|\sigma_{\Delta_{N}}\right\|_{2}^{2}
$$

and $\left\{\sigma_{\Delta_{N}}^{\prime \prime}\right\}$ is a Cauchy sequence in $L^{2}(a, b)$. As $L^{2}(a, b)$ is complete, there exists $y \in$ $L^{2}(a, b)$ such that

$$
\lim _{N \rightarrow \infty} \int_{a}^{b}\left|y(t)-\sigma_{\Delta_{N}}^{\prime \prime}(t)\right|^{2} d t=0
$$

Let us define $Y(t)=x^{\prime}(a)+\int_{a}^{t} y(u) d u$,

$$
\begin{aligned}
\left|Y(t)-\sigma_{\Delta_{N}}^{\prime}(t)\right| & \leq \int_{a}^{t}\left|y(u)-\sigma_{\Delta_{N}}^{\prime \prime}(u)\right| d u+\left|x^{\prime}(a)-\sigma_{\Delta_{N}}^{\prime}(a)\right| \\
& \leq\left\langle\left|y-\sigma_{\Delta_{N}}^{\prime \prime}\right|, 1\right\rangle_{L^{2}(a, b)}+\left|x^{\prime}(a)-\sigma_{\Delta_{N}}^{\prime}(a)\right| .
\end{aligned}
$$

Using Schwarz's inequality,

$$
\left|Y(t)-\sigma_{\Delta_{N}}^{\prime}(t)\right| \leq\left(\int_{a}^{b}\left|y(t)-\sigma_{\Delta_{N}}^{\prime \prime}(t)\right|^{2} d t\right)^{1 / 2}(b-a)^{1 / 2}+\left|x^{\prime}(a)-\sigma_{\Delta_{N}}^{\prime}(a)\right| .
$$

Considering (3.22), (3.24), and Theorem 3.4,

$$
\lim _{N \rightarrow \infty} \sigma_{\Delta_{N}}^{\prime}(t)=Y(t) .
$$


Besides,

$$
\left|x^{\prime}(t)-Y(t)\right| \leq\left|x^{\prime}(t)-\sigma_{\Delta_{N}}^{\prime}(t)\right|+\left|\sigma_{\Delta_{N}}^{\prime}(t)-Y(t)\right|
$$

Applying limits in this expression as $N \rightarrow \infty$, according to Theorem 3.4 and (3.25), $x^{\prime}=Y$ and $x^{\prime \prime}=Y^{\prime}=y$ almost everywhere. As a consequence by (3.22), as $N \rightarrow \infty$,

$$
\left\|\sigma_{\Delta_{N}}-x\right\|_{2} \longrightarrow 0
$$

and by (3.25),

$$
\left\|\sigma_{\Delta_{N}}^{\prime}-x^{\prime}\right\|_{\infty} \longrightarrow 0
$$

According to Theorem 3.4, as $\left\|\Delta_{N}\right\| \rightarrow 0$ and $x \in \mathscr{H}^{2}(a, b)$,

$$
\left\|\sigma_{\Delta_{N}}-x\right\|_{\infty} \longrightarrow 0
$$

The uniform convergence on $I=[a, b]$ implies $L^{2}$-convergence and

$$
\left\|\sigma_{\Delta_{N}}-x\right\|_{\mathscr{H}^{2}[a, b]} \longrightarrow 0
$$

Let us consider now any sequence of scaling factors such that $\left|s_{1}^{N}\right|<1 / N^{2+r}$, where $r>0$. One has

$$
\left\|x-\sigma_{\Delta_{N}}^{s^{N}}\right\|_{\mathscr{H}^{2}[a, b]} \leq\left\|x-\sigma_{\Delta_{N}}\right\|_{\mathscr{H}^{2}[a, b]}+\left\|\sigma_{\Delta_{N}}^{s^{N}}-\sigma_{\Delta_{N}}\right\|_{\mathscr{H}^{2}[a, b]} .
$$

Considering (2.44) and Lemma 3.8, for $j=0,1,2$,

$$
\left\|\left(\sigma_{\Delta_{N}}^{s^{N}}\right)^{(j)}-\sigma_{\Delta_{N}}^{(j)}\right\|_{L^{2}(a, b)} \leq \frac{N^{j}\left|s_{1}^{N}\right|}{1-N^{j}\left|s_{1}^{N}\right|}\left\|\sigma_{\Delta_{N}}^{(j)}-b^{(j)}\right\|_{L^{2}(a, b)} .
$$

As a consequence, bearing in mind that for $j=0,1,2$,

$$
\frac{N^{j}\left|s_{1}^{N}\right|}{1-N^{j}\left|s_{1}^{N}\right|} \leq \frac{N^{2}\left|s_{1}^{N}\right|}{1-N^{2}\left|s_{1}^{N}\right|}
$$

one has

$$
\left\|\sigma_{\Delta_{N}}^{s^{N}}-\sigma_{\Delta_{N}}\right\|_{\mathscr{H}^{2}[a, b]} \leq \frac{N^{2}\left|s_{1}^{N}\right|}{1-N^{2}\left|s_{1}^{N}\right|}\left\|\sigma_{\Delta_{N}}-b\right\|_{\mathscr{H}^{2}[a, b]}
$$

on the other hand,

$$
\left\|\sigma_{\Delta_{N}}-b\right\|_{\mathscr{H}^{2}[a, b]} \leq\left\|\sigma_{\Delta_{N}}-x\right\|_{\mathscr{H}^{2}[a, b]}+\|x-b\|_{\mathscr{H}^{2}[a, b]} .
$$

Due to the convergence of $\sigma_{\Delta_{N}}$ to $x$ in $\mathscr{H}^{2}[a, b]$ (3.30), $\left\|\sigma_{\Delta_{N}}-b\right\|_{\mathscr{H}^{2}[a, b]}$ is bounded (3.35). 
Since $\left|s_{1}^{N}\right|<1 / N^{2+r}$, taking limits in (3.34) as $N \rightarrow \infty$,

$$
\left\|\sigma_{\Delta_{N}}^{s^{N}}-\sigma_{\Delta_{N}}\right\|_{\mathscr{H}^{2}[a, b]} \longrightarrow 0 .
$$

Finally, by (3.30) and (3.31),

$$
\sigma_{\Delta_{N}}^{s^{N}} \longrightarrow x
$$

in $\mathscr{H}^{2}[a, b]$.

Consequence 3.10. The fractal cubic splines with non-null scale vector are dense in $\mathscr{H}^{2}[a, b]$.

Note. For $s^{N}=0$, we retrieve the standard spline case.

\subsection{Fractal cubic spline operator. Let}

$$
\Delta_{N}: a=t_{0}<t_{1}<t_{2}<\cdots<t_{N+1}=b
$$

be a given partition of the interval $[a, b]$. We follow here the spline definition of Laurent (see [9]). In order to simplify the notation, we will omit the subindex and we will write $\sigma(t)$ to represent a spline with respect to $\Delta_{N}$.

Definition 3.11. Let $S$ be the space of cubic spline functions $\sigma$ defined in $[a, b]$ such that

(i) $\sigma$ is a cubic polynomial on each subinterval $\left(t_{k}, t_{k+1}\right), k=1, \ldots, N-1$,

(ii) $\sigma$ is a polynomial of degree 1 in $\left[a, t_{1}\right)$ and $\left(t_{N}, b\right]$,

(iii) $\sigma^{\prime \prime}$ is continuous.

Note 1. $S$ is a linear subspace of $\mathscr{H}^{2}[a, b]$.

Note 2. In this case, $\sigma^{\prime \prime}(a)=\sigma^{\prime \prime}(b)=0$ (natural splines).

Note 3. Every $\sigma \in S$ can be expressed as (see [9]),

$$
\sigma(t)=\sum_{j=0}^{1} s_{j} t^{j}+\sum_{k=1}^{N} d_{k} \frac{\left(t-t_{k}\right)_{+}^{3}}{3 !},
$$

where

$$
\begin{aligned}
\left(t-t_{k}\right)_{+}^{3} & = \begin{cases}\left(t-t_{k}\right)^{3} & \text { if } t \geq t_{k}, \\
0 & \text { if } t<t_{k},\end{cases} \\
d_{k} & =\sigma^{(3)}\left(t_{k}+\right)-\sigma^{(3)}\left(t_{k}-\right) .
\end{aligned}
$$

Besides, $d_{k}$ satisfies the condition

$$
\sum_{k=1}^{N} d_{k}\left(t_{k}\right)^{K}=0, \quad K=0,1 .
$$


Let $\sigma_{k N}$ be the unique cubic spline defined by

$$
\sigma_{k N}\left(t_{j}\right)=\delta_{j k} \quad \forall j, k=1,2, \ldots, N
$$

where $\delta_{j k}$ is the delta of Kronecker. The family $\left\{\sigma_{k N}\right\}_{k=1}^{N}$ is an orthonormal system with respect to the form

$$
\langle f, g\rangle=\sum_{k=1}^{N} f\left(t_{k}\right) g\left(t_{k}\right) .
$$

Besides,

$$
S=\operatorname{span}\left\{\sigma_{k N}\right\}_{k=1}^{N}
$$

Let $s$ be a scale vector with constant coordinates such that $\left|s_{1}\right|<1 /(N+1)^{2}$. As $S \subset \mathscr{C}^{2}(I)$, we can consider the operator $\mathscr{D}_{p}^{s}$ defined in Section 2.4 for $p=2$. $\mathscr{D}_{2}^{s}(S)=S^{s}$ is the space of $s$-fractal functions of $S$ with respect to the partition

$$
\Delta_{N}: a=t_{0}<t_{1}<t_{2}<\cdots<t_{N+1}=b .
$$

Since $\mathscr{D}_{2}^{s}$ is linear,

$$
\mathscr{D}_{2}^{s}(S)=S^{s}=\operatorname{span}\left\{\sigma_{k N}^{s}\right\}_{k=1}^{N}
$$

The family $\sigma_{k N}^{s}$ is orthonormal respect to (3.43) because

$$
\sigma_{k N}^{s}\left(t_{j}\right)=\sigma_{k N}\left(t_{j}\right)=\delta_{j k}
$$

for $k, j=1,2, \ldots, N$. As a consequence, $\left\{\sigma_{k N}^{s}\right\}_{k=1}^{N}$ is a basis of $S^{s}$.

Let us define the fractal cubic spline operator with domain $\mathscr{C}[a, b]$ or, more specifically, $\mathscr{H}^{2}[a, b]$,

$$
\mathscr{Y}_{N}^{s}(g)(t)=\sum_{k=1}^{N} g\left(t_{k}\right) \sigma_{k N}^{s}(t) .
$$

Note that for all $k=1,2, \ldots, N$,

$$
S_{N}^{s}(g)\left(t_{k}\right)=g\left(t_{k}\right)
$$

since, for all $j, k=1,2, \ldots, N$,

$$
\sigma_{k N}^{s}\left(t_{j}\right)=\sigma_{k N}\left(t_{j}\right)=\delta_{j k}
$$

Proposition 3.12. $\mathscr{Y}_{N}^{s}$ is linear.

Proof. It is an immediate consequence of (3.48).

Without loss of generality, we consider $I=[0,1]$ and $t_{k}=k / N$. 
Lemma 3.13. Let $f$ and $g$ be defined in I such that $f\left(t_{k}\right)=g\left(t_{k}\right)$ for $k=1,2, \ldots, N$ for some $N$, then

$$
\mathscr{Y}_{N}^{s}(f)=\mathscr{Y}_{N}^{s}(g)
$$

Proof. For all $t \in I$,

$$
\mathscr{S}_{N}^{s}(f)(t)=\sum_{k=1}^{N} f\left(t_{k}\right) \sigma_{k N}^{s}(t)=\sum_{k=1}^{N} g\left(t_{k}\right) \sigma_{k N}^{s}(t)=\mathscr{Y}_{N}^{s}(g)(t) .
$$

Let us consider a sequence of partitions $\Delta_{N}$ such that $\Delta=\bigcup_{N \in \mathbb{N}} \Delta_{N}$ is dense in $I$.

LEMmA 3.14. If $f$ and $g$ are functions defined on I such that

$$
\lim _{N \rightarrow \infty} \mathscr{Y}_{N}^{s}(f)(t)=g(t) \quad \forall t \in \Delta,
$$

then

$$
f(t)=g(t) \quad \forall t \in \Delta
$$

Proof. If $t \in \Delta$, then $t \in \Delta_{N_{0}}$ for some $N_{0}, t=n / N_{0}$ and by (3.49),

$$
\mathscr{S}_{N_{0}}^{s}(f)(t)=f(t) .
$$

If the sequence of partitions $\left\{\Delta_{m N_{0}}\right\}$ is considered $t=n / N_{0}=m n / m N_{0} \in \Delta_{m N_{0}}$ and $\mathscr{S}_{m N_{0}}^{s}(f)(t)=f(t)$,

$$
f(t)=\lim _{m \rightarrow \infty} \mathscr{Y}_{m N_{0}}^{s}(f)(t)=g(t)
$$

Proposition 3.15. If, in addition to the hypotheses of Lemma 3.14, $f$ and $g \in \mathscr{H}^{2}[0,1]$, then $f=g$.

Proof. It is a consequence of the density of $\Delta$ in $I$ and the continuity of $f$ and $g$. If $t \in$ $[a, b]$ there exists $\bar{t}_{m} \in \Delta$ such that $\bar{t}_{m} \underset{m \rightarrow \infty}{\longrightarrow} t$ therefore, $f\left(\bar{t}_{m}\right) \rightarrow f(t)$ and $g\left(\bar{t}_{m}\right) \rightarrow g(t)$ as $m \rightarrow \infty$. But $f\left(\bar{t}_{m}\right)=g\left(\bar{t}_{m}\right)$ (previous lemma) and therefore, $f(t)=g(t)$, for all $t \in I$.

Proposition 3.16. If $g \in \mathscr{C}^{2}(I)$, then $\mathscr{Y}_{N}^{s}\left(g^{s}\right)=\mathscr{Y}_{N}^{s}(g)$. $\left(g^{s}=\mathscr{D}_{2}^{s}(g)\right.$ with respect to the partition $\Delta_{N}$.)

Proof. It is an immediate consequence of the definition of $\mathscr{Y}_{N}^{s}$ and the properties of interpolation of $g^{s}$ with respect to $g$.

Proposition 3.17. If $\sigma \in S$, then $\mathscr{Y}_{N}^{s}\left(\sigma^{s}\right)=\sigma^{s}$.

Proof. Let us consider $\sigma \in S$. By Proposition 3.16, since $\sigma \in \mathscr{C}^{2}(I)$,

$$
\mathscr{Y}_{N}^{s}\left(\sigma^{s}\right)(t)=\mathscr{Y}_{N}^{s}(\sigma)(t)=\sum_{k=1}^{N} \sigma\left(t_{k}\right) \sigma_{k N}^{s}(t)
$$


On the other hand,

$$
\sigma(t)=\sum_{k=1}^{N} \sigma\left(t_{k}\right) \sigma_{k N}(t)
$$

because the spline passing through $\sigma\left(t_{k}\right)$ is unique. By the linearity of $\mathscr{D}_{2}^{s}$,

$$
\sigma^{s}(t)=\sum_{k=1}^{N} \sigma\left(t_{k}\right) \sigma_{k N}^{s}(t),
$$

by (3.57) and (3.59),

$$
\mathscr{S}_{N}^{s}\left(\sigma^{s}\right)=\sigma^{s}
$$

Consequence 3.18. The fractal cubic splines with scale vector $s$ are eigenfunctions (or fixed points) of the operator $\mathscr{Y}_{N}^{s}$.

Proposition 3.19. $\mathscr{Y}_{N}^{s}$ is a projector of $\mathscr{H}^{2}[0,1]$,

$$
\mathscr{Y}_{N}^{s} \circ \mathscr{Y}_{N}^{s}=\mathscr{Y}_{N}^{s} \text {. }
$$

Proof. For all $g \in \mathscr{H}^{2}[0,1]$,

$$
\mathscr{Y}_{N}^{s}(g)=\sum_{k=1}^{N} g\left(t_{k}\right) \sigma_{k N}^{s} \in S^{s}
$$

and, as a consequence, $\mathscr{Y}_{N}^{s}(g)$ is a fixed point of $\mathscr{Y}_{N}^{s}$ (Proposition 3.17),

$$
\mathscr{S}_{N}^{s}\left(\mathscr{Y}_{N}^{s}(g)\right)=\mathscr{Y}_{N}^{s}(g)
$$

\section{References}

[1] J. H. Ahlberg, E. N. Nilson, and J. L. Walsh, The Theory of Splines and Their Applications, Academic Press, New York, 1967.

[2] M. F. Barnsley, Fractal functions and interpolation, Constructive Approximation 2 (1986), no. 4, 303-329.

[3] _ Fractals Everywhere, Academic Press, Massachusetts, 1988.

[4] M. F. Barnsley and A. N. Harrington, The calculus of fractal interpolation functions, Journal of Approximation Theory 57 (1989), no. 1, 14-34.

[5] A. K. B. Chand and G. P. Kapoor, Generalized cubic spline fractal interpolation functions, SIAM Journal on Numerical Analysis 44 (2006), no. 2, 655-676.

[6] P. G. Ciarlet, M. H. Schultz, and R. S. Varga, Numerical methods of high-order accuracy for nonlinear boundary value problems. I. One dimensional problem, Numerische Mathematik 9 (1967), no. 5, 394-430.

[7] C. A. Hall and W. W. Meyer, Optimal error bounds for cubic spline interpolation, Journal of Approximation Theory 16 (1976), no. 2, 105-122.

[8] J. E. Hutchinson, Fractals and self-similarity, Indiana University Mathematics Journal 30 (1981), no. $5,713-747$. 


\section{Smooth fractal interpolation}

[9] P.-J. Laurent, Approximation et optimisation, Hermann, Paris, 1972.

[10] M. A. Navascués and M. V. Sebastián, Some results of convergence of cubic spline fractal interpolation functions, Fractals 11 (2003), no. 1, 1-7.

[11] Fitting curves by fractal interpolation: an application to the quantification of cognitive brain processes, Thinking in Patterns: Fractals and Related Phenomena in Nature (M. M. Novak, ed.), World Scientific, New Jersey, 2004, pp. 143-154.

[12] Generalization of Hermite functions by fractal interpolation, Journal of Approximation Theory 131 (2004), no. 1, 19-29.

[13] , A fractal version of Schultz's theorem, Mathematical Inequalities \& Applications 8 (2005), no. 1, 63-70.

[14] J. Stoer and R. Bulirsch, Introduction to Numerical Analysis, Springer, New York, 1980.

M. A. Navascués: Departamento de Matemática Aplicada, Universidad de Zaragoza, C/María de Luna 3, Zaragoza 50018, Spain

E-mail address: manavas@unizar.es

M. V. Sebastián: Departamento de Matemáticas, Universidad de Zaragoza,

Campus Plaza de San Francisco s/n, Zaragoza 50009, Spain

E-mail address: msebasti@unizar.es 